\title{
The clinical application of enhanced recovery after surgery protocol in perioperative period of laparoscopic nephron sparing surgery
}

\author{
Min Tang", Huiyu Dong", Pengfei Shao, Jie Li, Zengjun Wang, Bianjiang Liu \\ Department of Urology, The First Affiliated Hospital of Nanjing Medical University, Nanjing, China \\ Contributions: (I) Conception and design: M Tang, B Liu; (II) Administrative support: Z Wang; (III) Provision of study materials or patients: H Dong; \\ (IV) Collection and assembly of data: P Shao; (V) Data analysis and interpretation: J Li; (VI) Manuscript writing: All authors; (VII) Final approval of \\ manuscript: All authors. \\ \#These authors contributed equally to this study. \\ Correspondence to: Bianjiang Liu. Department of Urology, The First Affiliated Hospital of Nanjing Medical University, 300 Guangzhou Road, \\ Nanjing, China. Email: bjliu@njmu.edu.cn.
}

\begin{abstract}
Background: The enhanced recovery after surgery (ERAS) program is focused on improving surgical outcomes and enhancing the patient experience before, during and after surgery. We performed this study to evaluate the effect of ERAS in perioperative period of laparoscopic nephron sparing surgery (LNSS).

Methods: A retrospective analysis of 287 consecutive patients with localized renal cell carcinoma (RCC) who underwent LNSS from December 2015 to June 2017 was conducted. Our study design included two cohorts. Patients underwent conventional care in one group ( $\mathrm{n}=136)$, and ERAS protocol in the other group $(\mathrm{n}=151)$. The data of recovery of gastrointestinal function, catheter and drainage tube removal time, length of stay (LOS), hospitalization expenses and incidence of postoperative complications were compared between the two groups.

Results: Compared with conventional group, cases in ERAS group presented with shorter time of recovery of gastrointestinal function $(\mathrm{P}<0.05)$ and $\operatorname{LOS}(\mathrm{P}<0.05)$, shorter catheter and drainage tube removal time $(\mathrm{P}<0.05)$, lower hospitalization expenses and lower perioperative complications $(\mathrm{P}<0.05)$.

Conclusions: Program of ERAS used in perioperative period of LNSS can apparently accelerate the time of postoperative recovery, reduce complications, shorten time stay in hospital and cut down the total cost, finally improve patients' satisfaction.
\end{abstract}

Keywords: Enhanced recovery after surgery (ERAS); laparoscopic nephron-sparing surgery (LNSS); renal cell carcinoma (RCC)

Submitted Dec 27, 2019. Accepted for publication Jun 09, 2020.

doi: $10.21037 /$ tau-19-924

View this article at: http://dx.doi.org/10.21037/tau-19-924

\section{Introduction}

Laparoscopic nephron-sparing surgery (LNSS) has been widely accepted as an effective way to treat $\mathrm{T} 1$ renal cell carcinoma (RCC) (1). Nowadays, LNSS has been greatly improved, especially the novel technique of laparoscopic partial nephrectomy with precise segmental renal artery clamping widely adopted in our center $(1,2)$. The enhanced recovery after surgery (ERAS) represents a multidisciplinary collaboration which would transform traditional care programs into an effective postoperative rehabilitation program, in order to realize a shorter hospitalization, reduced complications and a reduction of medical cost with increased satisfaction and safety after discharge (3). To date, no studies have shown whether the ERAS protocol is superior to conventional method after LNSS.

In this study, we retrospectively compared postoperative 
outcomes and perioperative complications of patients with RCC after the ERAS or conventional protocols between December 2015 and March 2017. We present the following article in accordance with the STROBE reporting checklist (available at http://dx.doi.org/10.21037/tau-19-924).

\section{Methods}

\section{Ethics statement}

Approval for this study was granted by the ethics committee of Nanjing Medical University (China) (NO.:2015SRFA-042) and informed written consent was received from all participants. All research procedures were conducted in accordance with the Declaration of Helsinki (as revised in 2013).

\section{Clinical materials}

A retrospective analysis of 287 consecutive patients with localized RCC who underwent LNSS with precise segmental renal artery at our department between December 2015 and March 2017 was performed. Inclusion criteria: Renal cancer diagnosed by pathology; R.E.N.A.L. nephrometry score (RNS) $\leq 10$; Body mass index (BMI) $<35$; ASA score $\leq 3$; clear minded; fluency in expressing; the patient or his agent is informed of this study. Exclusion criteria: $\mathrm{RNS}>10$; $\mathrm{BMI} \geq 35$; ASA score $>3$; mental anomaly; combined with severe cardiovascular disease, nervous system disease or motor system disease. The effects on perioperative outcomes were examined by comparing 136 consecutive patients before introduction of the ERAS protocol (conventional group) with 151 consecutive patients after introduction of the ERAS protocol (ERAS group).

The principle of conventional care and the ERAS protocol were shown in Table 1.

\section{Protocols of ERAS and conventional groups}

The variables that were recorded included the recovery time of gastrointestinal function, removing time of catheter and drainage tube, postoperative hospital stay, hospitalization expense and complications. Postoperative recovery of gastrointestinal function was evaluated as recovery time of bowel sound, first exhaust time and first defecation time. Perioperative complications were assessed as preoperative hypoglycemia, postoperative chills and fever, postoperative hypostatic pneumonia, postoperative abdominal distention, postoperative stress ulcer, postoperative cardiac cerebrovascular accident, incision fat liquefaction, urinary system infection and venous thrombosis.

\section{Statistical analysis}

Statistical analyses were performed with SPSS 13.0 software. Student's $t$-test and the Chi-square test were used to analyze the differences between groups. $\mathrm{P}<0.05$ was considered to indicate a statistical significant difference.

\section{Results}

\section{Patients' characteristics}

As shown in Table 2, there was no statistical significance about the demographics and tumor characteristics of patients between two groups $(\mathrm{P}>0.05)$.

\section{Intraoperative data and Postoperative outcomes}

No significant differences were observed in operation time \{ERAS group 85 [50-122] min vs. conventional group 82 [55-130] min, $\mathrm{P}>0.05\}$, and blood loss \{ERAS group 200 [10-600] $\mathrm{mL} v s$. conventional group 180 [5-500] mL, $\mathrm{P}>0.05$ \} between both the groups.

As shown in Table 3, the ERAS group had marked shorter time to the recovery of gastrointestinal function (recovery time of bowel sounds, first exhaust time and first defecation time) than did the conventional group $(\mathrm{P}<0.01)$. Compared with conventional group, the ERAS group also showed shorter time of removing catheter $(\mathrm{P}<0.01)$ and drainage tube $(\mathrm{P}<0.01)$, shorter postoperative hospital stay $(\mathrm{P}<0.01)$ and lower hospitalization expenses $(\mathrm{P}<0.01)$.

\section{Perioperative complications}

Patients in both groups were discharged successfully and there was no death case. As shown in Table 4, there were only 5 cases of complications in ERAS group, which was significantly less than that of conventional group ( 36 cases, $\mathrm{P}<0.01$ ).

\section{Discussion}

The ERAS protocol is standardized, multidisciplinary and evidence-based tools that provide guidelines for clinical decision-making for enhancing recovery of patients after surgery (4). The key goals of ERAS protocol are to reduce 
Table 1 Differences of perioperative management methods between ERAS and conventional groups

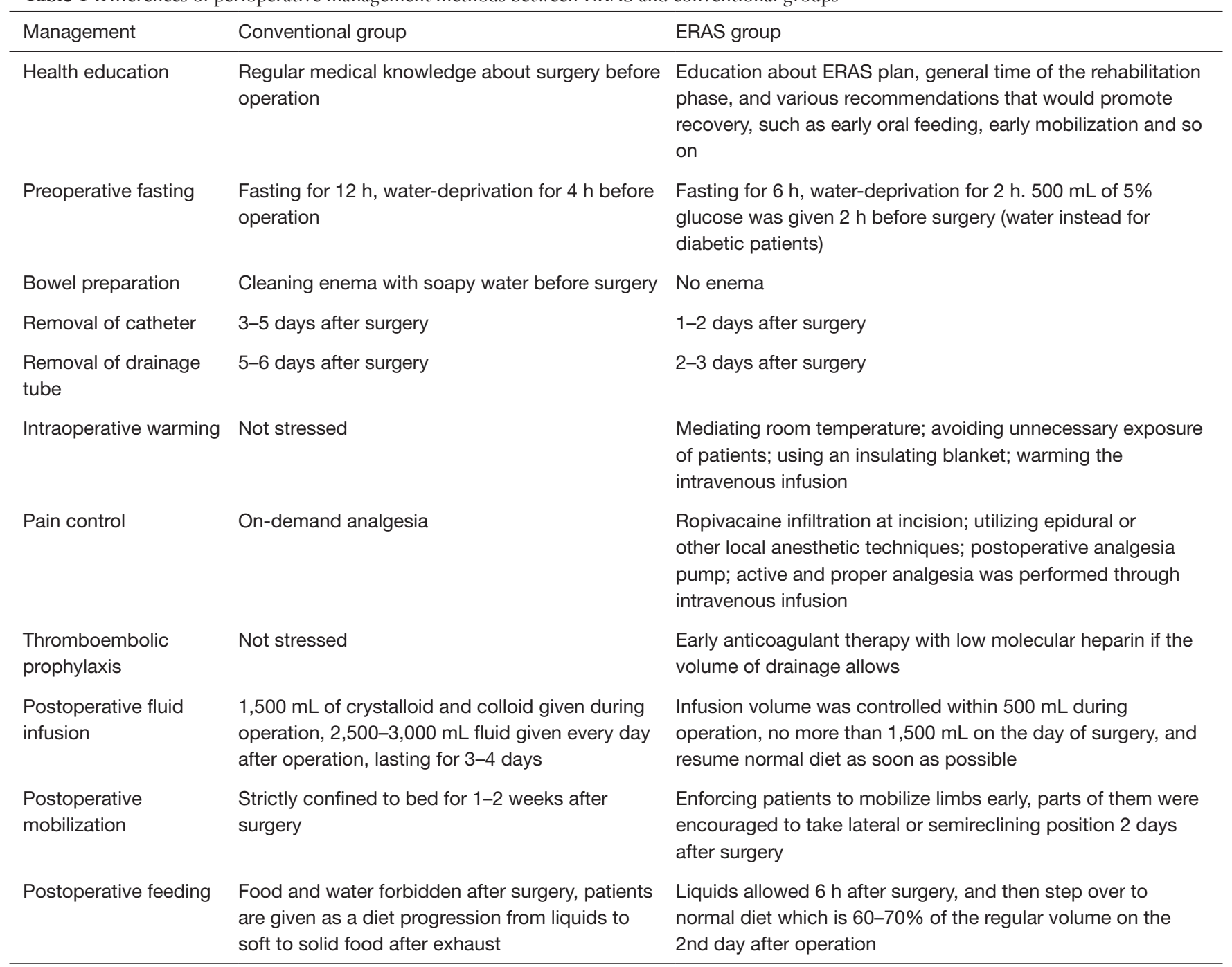

ERAS, enhanced recovery after surgery.

postoperative complications, shorten hospitalization and cut medical cost as well as enhance safety. In this study, a multidisciplinary ERAS program for LNSS was implemented at our center. Eventually, postoperative recovery was apparently accelerated, complications were reduced, time stay in hospital was shortened and the total cost was obviously cut down. Several pivotal items among ERAS concept have played a crucial role in enhanced recovery after LNSS. First of all, clear fluid should be allowed up to $2 \mathrm{~h}$ and solids up to $6 \mathrm{~h}$ before induction of anesthesia. Carbohydrate should be used regularly up to $2 \mathrm{~h}$ prior to surgery. Secondly, mechanical bowel preparation should be abandoned before renal operation. Thirdly, administering too much fluid during or after surgery might result in bowel enema, pulmonary edema, water intoxication and so on, all of which would prolong the recovery. Thus, intraoperative and postoperative restricted fluid therapy should be used. In addition, early removal of catheter and drainage tube as well as satisfactory pain control can promote early mobilization, which can also reduce complications as hypostatic pneumonia and venous thrombosis.

Recovery of gastrointestinal function is an important indicator of postoperative recovery. The ERAS protocol suggests avoiding of preoperative mechanical enema, preoperative carbohydrate loading and restricted fluid therapy, which have been strictly performed in our ERAS group. It was reported that the time of first flatus was shorter in fast-track laparoscopic retroperitoneal 
Table 2 Patient demographics and tumor characteristics

\begin{tabular}{|c|c|c|c|c|}
\hline Category & ERAS $(n=151)$ & Conventional $(n=136)$ & $\chi^{2} / \mathrm{t}$ value & $P$ value \\
\hline Age, years & $56.1 \pm 3.5$ & $54.7 \pm 4.3$ & 1.65 & 0.05 \\
\hline RNS & $6.1 \pm 1.7$ & $5.9 \pm 1.6$ & 1.02 & 0.15 \\
\hline BMI & $23.2 \pm 5.1$ & $22.3 \pm 4.7$ & 1.56 & 0.06 \\
\hline Tumor side & & & 2.84 & 0.09 \\
\hline Left & 73 & 78 & & \\
\hline Right & 78 & 58 & & \\
\hline Tumor location & & & 0.07 & 0.79 \\
\hline Lower pole & 58 & 55 & & \\
\hline Tumor size, $\mathrm{cm}$ & $2.6 \pm 0.9$ & $2.8 \pm 1.3$ & -1.23 & 0.11 \\
\hline
\end{tabular}

ERAS, enhanced recovery after surgery.

Table 3 Comparison of postoperative outcomes between conventional and ERAS groups

\begin{tabular}{|c|c|c|c|c|}
\hline Variable & Conventional & ERAS & $\mathrm{t}$ & $\mathrm{P}$ \\
\hline Recovery time of bowel sounds (h) & $27.3 \pm 2.6$ & $21.1 \pm 3.6$ & 18.03 & $<0.01$ \\
\hline First exhaust time (h) & $47.3 \pm 9.2$ & $33.6 \pm 8.9$ & 12.93 & $<0.01$ \\
\hline First defecation time (h) & $71.3 \pm 9.6$ & $55.4 \pm 9.2$ & 11.26 & $<0.01$ \\
\hline Time of removing drainage tube $(\mathrm{d})$ & $4.6 \pm 0.7$ & $3.2 \pm 1.1$ & 9.15 & $<0.01$ \\
\hline Postoperative hospital stay (d) & $8.1 \pm 1.6$ & $4.6 \pm 1.7$ & 14.86 & $<0.01$ \\
\hline Hospitalization expenses (ten thousand yuan) & $5.1 \pm 0.3$ & $4.3 \pm 0.1$ & 21.71 & $<0.01$ \\
\hline
\end{tabular}

ERAS, enhanced recovery after surgery.

adrenalectomy compared with the conventional protocol (5). In our study, the ERAS group had significantly shorter time to the recovery of gastrointestinal function than did the conventional group. Besides, enforced postoperative early mobilization might be another important factor for fast recovery of gastrointestinal function (6). Thus, patients in ERAS group were enforced to mobilize limbs early, parts of whom were encouraged to take lateral or semireclining position 2 days after LNSS, as long as suture was reliable. In addition, postoperative early oral feedings can promote intestinal peristalsis, maintain the function of intestinal mucosa, prevent bacterial translocation, and help patients to recover as soon as possible (7). In this study, a liquid diet was recommended for patients $6 \mathrm{~h}$ after operation in ERAS group. And if there was no nausea, vomiting, or ileus, normal eating can resume 3 days after surgery.

In the current study, there were significant differences of several complications between both groups. Among these, lower incidence of preoperative hypoglycemia in ERAS group might be attributed to a regular diet until 
Table 4 Comparison of perioperative complications between ERAS and conventional groups

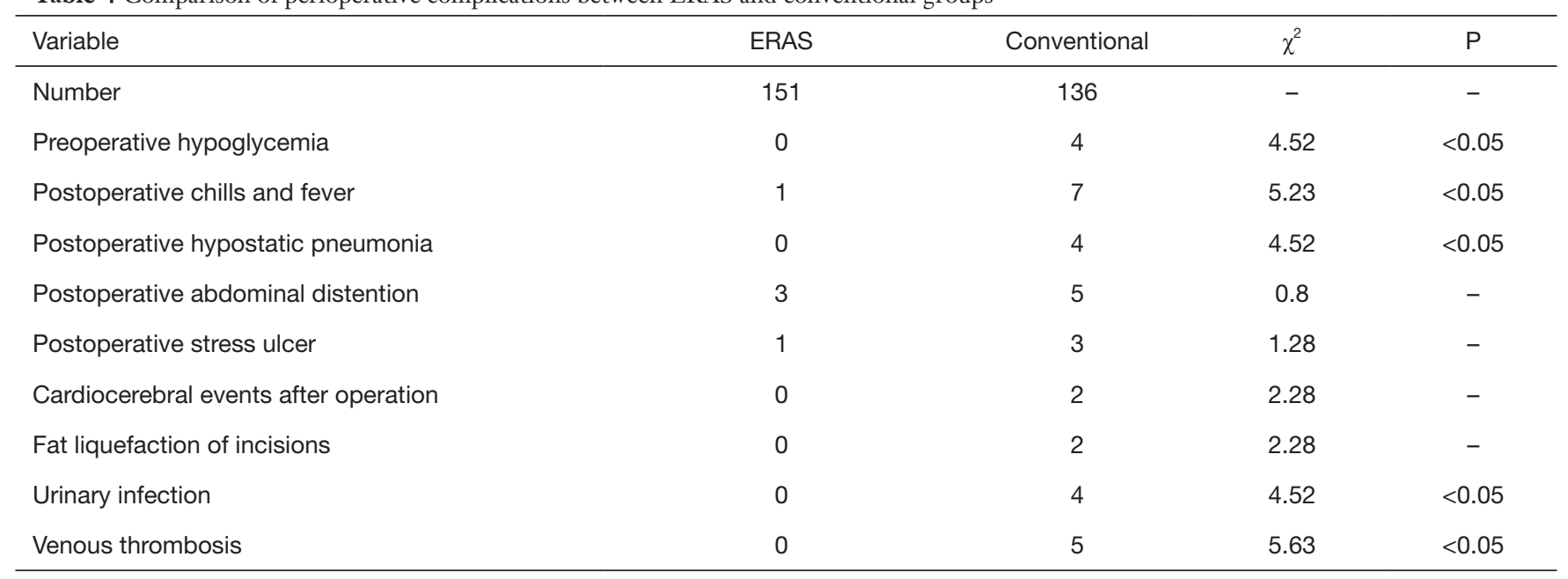

ERAS, enhanced recovery after surgery.

6 hours before surgery and a drink of $500 \mathrm{~mL} \mathrm{5 \%} \mathrm{glucose}$ 2 hours before operation. Recent clinical studies have shown that preoperative intake of a carbohydrate drink may also reduce the postoperative endocrine catabolic responses and improve insulin resistance (8). Secondly, low rate of postoperative hypostatic pneumonia may be due to early postoperative mobilization and restricted fluid therapy. Finally, there was an obvious lower incidence of venous thrombosis than did the conventional group. In ERAS group of this study, low molecular-weight heparin has been administered as soon as the volume of drainage is less than $50 \mathrm{~mL}$, maintained to discharge. ASCO guideline has recommended most patients with active cancers to receive prophylactic treatment of thrombus throughout the hospital stay. It seems that regular thromboembolic prophylaxis after LNSS has significant clinical value.

To the best of our knowledge, this is the first study to introduce the ERAS protocol for LNSS. However, there were several limitations of this study. Firstly, this study was not a randomized study and was a single centre retrospective study. Besides, the size of sample population was simply based on the number of patients enrolled at our department, which was not specifically calculated according to a study design. Additionally, the management of protocol might be slightly changed due to subjective factor from patients, which might result in inevitable bias of results.

\section{Conclusions}

A comprehensive revision of postoperative care called
ERAS in patients, who accepted LNSS, may accelerate rehabilitation, decrease complications, cut down hospitalization expenses and increase satisfaction of patients. But implementation of the ERAS protocol is especially difficult when only performed in urology department. Successful application of the ERAS protocol depends on close cooperation with anesthesiologists, nurses, physiotherapy staff, patients and their family and so on.

\section{Acknowledgments}

Funding: This work was supported by the grant from National Natural Science Foundation of China (81600514), Six Talent Peak Project of High-level Talents in Jiangsu Province (WSW-017), 333 High-level Talents Training Project in Jiangsu Province, Professional from Six-Pronged Top-Talent Program (LGY2018053), Qing Lan Project of Jiangsu University (JX2161015100), The Fifth Batch of Outstanding Young and Middle-aged Teachers' Support Program of Nanjing Medical University, and A Project Funded by the Priority Academic Program Development of Jiangsu Higher Education Institutions (JX10231802).

\section{Footnote}

Reporting Checklist: The authors have completed the STROBE reporting checklist. Available at http://dx.doi. org/10.21037/tau-19-924

Data Sharing Statement: Available at http://dx.doi. 
org/10.21037/tau-19-924

Conflicts of Interest: All authors have completed the ICMJE uniform disclosure form (available at http://dx.doi. org/10.21037/tau-19-924). The authors have no conflicts of interest to declare.

Ethical Statement: The authors are accountable for all aspects of the work in ensuring that questions related to the accuracy or integrity of any part of the work are appropriately investigated and resolved. Approval for this study was granted by the ethics committee of Nanjing Medical University (China) (NO.:2015-SRFA-042) and informed written consent was received from all participants. All research procedures were conducted in accordance with the Declaration of Helsinki (as revised in 2013).

Open Access Statement: This is an Open Access article distributed in accordance with the Creative Commons Attribution-NonCommercial-NoDerivs 4.0 International License (CC BY-NC-ND 4.0), which permits the noncommercial replication and distribution of the article with the strict proviso that no changes or edits are made and the original work is properly cited (including links to both the formal publication through the relevant DOI and the license). See: https://creativecommons.org/licenses/by-nc-nd/4.0/.

Cite this article as: Tang $M$, Dong H, Shao P, Li J, Wang Z, Liu B. The clinical application of enhanced recovery after surgery protocol in perioperative period of laparoscopic nephron sparing surgery. Transl Androl Urol 2020;9(4):1566-1571. doi: 10.21037/tau-19-924

\section{References}

1. Shao P, Qin C, Yin C, et al. Laparoscopic partial nephrectomy with segmental renal artery clamping: technique and clinical outcomes. Eur Urol 2011;59:849-55.

2. Shao P, Tang L, Li P, et al. Precise segmental renal artery clamping under the guidance of dual-source computed tomography angiography during laparoscopic partial nephrectomy. Eur Urol 2012;62:1001-8.

3. Kehlet H, Wilmore DW. Multimodal strategies to improve surgical outcome. Am J Surg 2002;183:630-41.

4. Jiang ZW, Li JS. Fast Track Surgery: Optimized Clinical Pathways. Zhonghua Wei Chang Wai Ke Za Zhi 2012;15:12-3.

5. Tang C, Xu Z, Yi X, et al. Fast track surgery vs. conventional management in the perioperative care of retroperitoneal laparoscopic adrenalectomy. Int J Clin Exp Med 2015;8:16207-14.

6. Agnelli G. Prevention of venous thromboembolism in surgical patients. Circulation 2004;110:IV4-IV12.

7. Osland E, Yunus RM, Khan S, et al. Early versus traditional postoperative feeding in patients undergoing resectional gastrointestinal surgery: a meta-analysis. JPEN J Parenter Enteral Nutr. 2011;35:473-87.

8. Moro ET. Prevention of pulmonary gastric contents aspiration. Rev Bras Anestesiol 2004;54:261-75. 